\title{
Low-frequency bursts of horizontally polarized waves in the Arctic sea-ice cover
}

\author{
David MARSAN, ${ }^{1}$ Jérôme WEISS, ${ }^{2}$ Jean-Philippe MÉTAXIAN, ${ }^{1,3}$ \\ Jacques GRANGEON, ${ }^{1}$ Pierre-François ROUX, ${ }^{1}$ Jari HAAPALA ${ }^{4}$ \\ ${ }^{1}$ Laboratoire de Géophysique Interne et Tectonophysique, Université de Savoie, 77376 Le-Bourget-du-Lac, France \\ E-mail: david.marsan@univ-savoie.fr \\ ${ }^{2}$ Laboratoire de Glaciologie et Géophysique de l'Environnement, CNRS/Université Joseph Fourier - Grenoble I, \\ 54 rue Molière, BP 96, 38402 Saint-Martin-d'Hères Cedex, France \\ ${ }^{3}$ Institut de Recherche pour le Développement, 213 rue La Fayette, 75480 Paris Cedex 10, France \\ ${ }^{4}$ Finnish Institute of Marine Research, PO Box 33, FIN-00931 Helsinki, Finland
}

\begin{abstract}
We report the detection of bursts of low-frequency waves, typically $f=0.025 \mathrm{~Hz}$, on horizontal channels of broadband seismometers deployed on the Arctic sea-ice cover during the DAMOCLES (Developing Arctic Modeling and Observing Capabilities for Long-term Environmental Studies) experiment in spring 2007. These bursts have amplitudes well above the ambient ice swell and a lower frequency content. Their typical duration is of the order of minutes. They occur at irregular times, with periods of relative quietness alternating with periods of strong activity. A significant correlation between the rate of burst occurrences and the ice-cover deformation at the $\sim 400 \mathrm{~km}$ scale centered on the seismic network suggests that these bursts are caused by remote, episodic deformation involving shearing across regional-scale leads. This observation opens the possibility of complementing satellite measurements of ice-cover deformation, by providing a much more precise temporal sampling, hence a better characterization of the processes involved during these deformation events.
\end{abstract}

\section{INTRODUCTION}

The Arctic sea-ice cover is subject to many vibrational processes, either quasi-permanent or transient, that take the form of mechanical waves traveling within the ice. Ocean surface waves are the most common mechanism for generating waves in pack ice, in particular close to the ice edge (Squire, 2007), but long infra-gravity waves penetrate thousands of kilometers inside the ice cover (Squire and others, 2009; Wadhams and Doble, 2009). In addition to externally forced motion, other types of waves are also observed in the pack due to its kinematics, including fracturing, ridge build-up and ice floe/floe collision (Yang and Giellis, 1994; Dudko, 1999).

In situ observations of waves in the Arctic ice cover have mostly focused on understanding the origin of the Arctic ambient noise, generally recorded with hydrophones located at various depths in the water column (Milne and Ganton, 1964; Makris and Dyer, 1986; Greening and Zakarauskas, 1994) and on characterizing the propagation characteristics (wave speed and attenuation) of the different vibration modes in the ice cover (Hunkins, 1960; Stein, 1988; Xie and Farmer, 1994; Stein and others, 1998; Dosso and others, 2002), including edge waves propagating along the edges of a lead (Dudko and others, 1998). The frequency band typically probed by these analyses ranges from $1 \mathrm{~Hz}$ to several hundred $\mathrm{Hz}$, which corresponds to the frequencies excited by and transmitted from small cracks over relatively short distances (up to a few kilometers), including thermal cracks (Crary, 1955; Milne and others, 1967; Xie and Farmer, 1994). These impulsive 'ice transients' are ubiquitous and can be investigated in detail using compact seismic arrays, with limited (up to kilometers) apertures. Crack lengths of a few meters, associated with sub-millimeter displacements, have been reported (Dudko, 1999). These high-frequency events are very different from the large-scale shear motions localized along leads, which accommodate most sea-ice brittle deformation.

In comparison, lower-frequency waves in ice have attracted less attention, possibly for two distinct reasons: the frequency cut-off of geophones and hydrophones is generally $\sim 1 \mathrm{~Hz}$; moreover, at low frequencies (periods peaking at about 20-30s), the continuously activated ice swell dominates the signal. No ice transients have been found at such low frequencies, with the notable exception of Yang and Giellis (1994) who identified horizontally polarized low-frequency $(f<9 \mathrm{~Hz}$ ) transients that they attributed to the bumping and rubbing of the ice floe on which the geophones were deployed against a neighboring floe. Also, LeSchack and Haubrich (1964) report an increase in the spectral density of vertical motion for periods of up to $100 \mathrm{~s}$ during episodes of high winds; they did not measure horizontal oscillation as they were using gravity meters.

Here we report for the first time that, in addition to the well-known infra-gravity waves and high-frequency vibrations of the ice cover, there also exist ice transients with peak periods ranging from 30 to $50 \mathrm{~s}$, and typical durations of a few to tens of minutes. These transients take the form of very clear, emergent low-frequency bursts (LFBs), with amplitudes well above the amplitude of the ambient ice swell. We first describe their general characteristics, then go on to discuss their possible origins.

\section{DATA}

A seismic network was deployed in April 2007 as part of the measurement campaign at the Tara drifting station operating in the framework of the DAMOCLES (Developing Arctic Modeling and Observing Capabilities for 


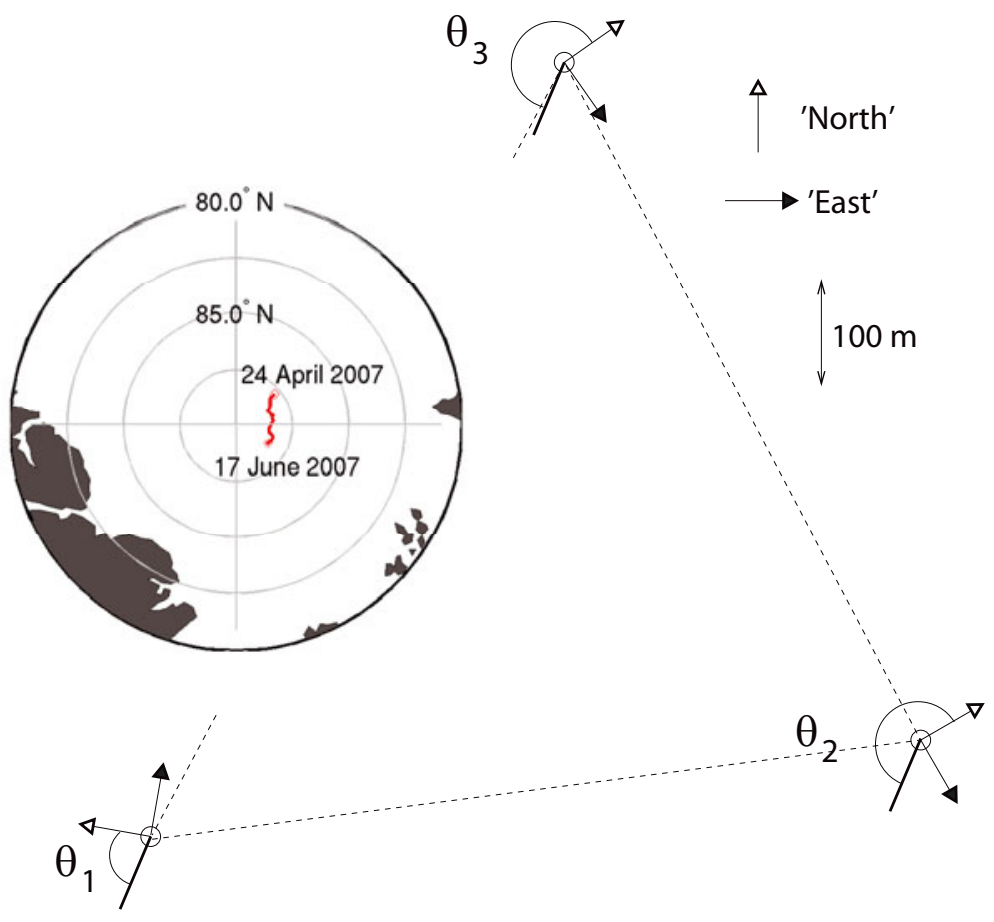

Fig. 1. Network configuration and its drifting trajectory between 24 April and 17 June 2007 (insert). The angles, $\theta_{1}, \theta_{2}$ and $\theta_{3}$, between the north channels and a given arbitrary direction (thick segment) are unknown. A correlation analysis that maximizes the cross-correlation between linear combinations of the two horizontal channels at the three stations is used to estimate the relative angles, $\theta_{1}-\theta_{2}, \theta_{1}-\theta_{3}$ and $\theta_{2}-\theta_{3}$, with good accuracy.

Long-term Environmental Studies) project (Gascard and others, 2008). From this network made of 16 short-period $(1 \mathrm{~Hz})$ vertical seismometers and 5 broadband Güralp CMG3ESPC seismometers, we here only use data recorded by three of the latter instruments. Data from the two remaining CMG-3ESPC could not be exploited; one had acquisition problems, while the other was located on a neighboring floe, and recorded a signal that is too different from the others to be used in this analysis. The broadband seismometers have a 60 s low-frequency cut-off, and their signals were sampled at $100 \mathrm{~Hz}$. The stations were placed at distances of 620-740 m from each other (Fig. 1). As shown below, the signals at these three stations are well correlated. We analyze data acquired between 24 April and 17 June 2007. Although the network operated until September, the quality of the data becomes questionable after 17 June 2007. The network drifted along with the Tara base camp at roughly constant latitude $\left(88^{\circ} 14^{\prime}-\right.$ $88^{\circ} 32^{\prime} \mathrm{N}$; see Fig. 1). No detectable deformation occurred within this network during this time period.

At the time of deployment, the absolute angles at which the horizontal channels are aligned were unfortunately not correctly reported. We were able to reconstruct, a posteriori, the relative angles between the three stations, by maximizing the correlation between rotated horizontal waveforms. More precisely, each station records the displacement rates, a $(t)$ and $b(t)$, of the ice along two orthogonal directions, on top of the vertical displacement rate. The horizontal displacement rate, $h(t)$, along a direction oriented at angle $\phi$ relative to the direction of the first channel, $a(t)$, is obtained using $h(t)=a(t) \cos \phi+b(t) \sin \phi$. For two stations 1 and 2 , the relative angle, $\Delta \theta=\theta_{2}-\theta_{1}$, of their first channels, $a_{1}(t)$ and $a_{2}(t)$, is determined by maximizing the correlation between $h_{1}(t)=a_{1}(t) \cos \phi+b_{1}(t) \sin \phi$ and $h_{2}(t)=a_{2}(t) \cos (\phi+\Delta \theta)+$ $b_{2}(t) \sin (\phi+\Delta \theta)$, whatever the value of $\phi$. Hence, denoting by $\theta_{1}, \theta_{2}$ and $\theta_{3}$ the angles of the first horizontal channel of the three stations relative to a given direction (e.g. 'true' north; Fig. 1), we obtain with good accuracy $\left(\sim 2^{\circ}\right)$ the differences $\theta_{1}-\theta_{2}, \theta_{1}-\theta_{3}$ and $\theta_{2}-\theta_{3}$. This allows us to relatively align the horizontal traces at the three stations so they all point in a common direction, even though we do not know how this direction is oriented relative to the seismic network.

In order to relate the occurrence of wave transients, as described in the next section, to ice-cover deformation transients, we used the positions of the 16 drifting ARGOS (Advanced Research and Global Observation Satellite) buoys deployed by the Meteorological Institute of Hamburg, as part of the DAMOCLES experiment. These buoys were initially deployed to form a $400 \mathrm{~km}$ long square centered on Tara.

\section{DETECTION AND CHARACTERIZATION OF LOW-FREQUENCY BURSTS}

\section{Detection}

The broadband signals are mostly dominated by the ice swell, which has a peak period ranging from 20 to $30 \mathrm{~s}$. The ice swell manifests itself with equal amplitude on the vertical and horizontal channels. Figure 2a shows 1 hour of recording that only contains the ice-swell signal, which at this time has a peak period of $27 \mathrm{~s}$. Peak-to-peak amplitude is $\sim 0.1 \mathrm{~mm} \mathrm{~s}^{-1}$, which corresponds to $\sim 0.5 \mathrm{~mm}$ of peakto-peak vertical and horizontal displacement, as is typically observed (e.g. Hunkins, 1962; LeSchack and Haubrich, 1964; Dugan and others, 1992). In contrast, LFBs can be seen at specific times on the horizontal channels only, and are characterized by much greater amplitudes. Figure 2c shows such a LFB, characterized by a finite duration $(7 \mathrm{~min})$, and a clear increase in the energy content at a period $>27 \mathrm{~s}$ (Fig. 2d). Similar signals, with cross-correlation $>95 \%$, are observed at all three stations, which excludes the possibility 

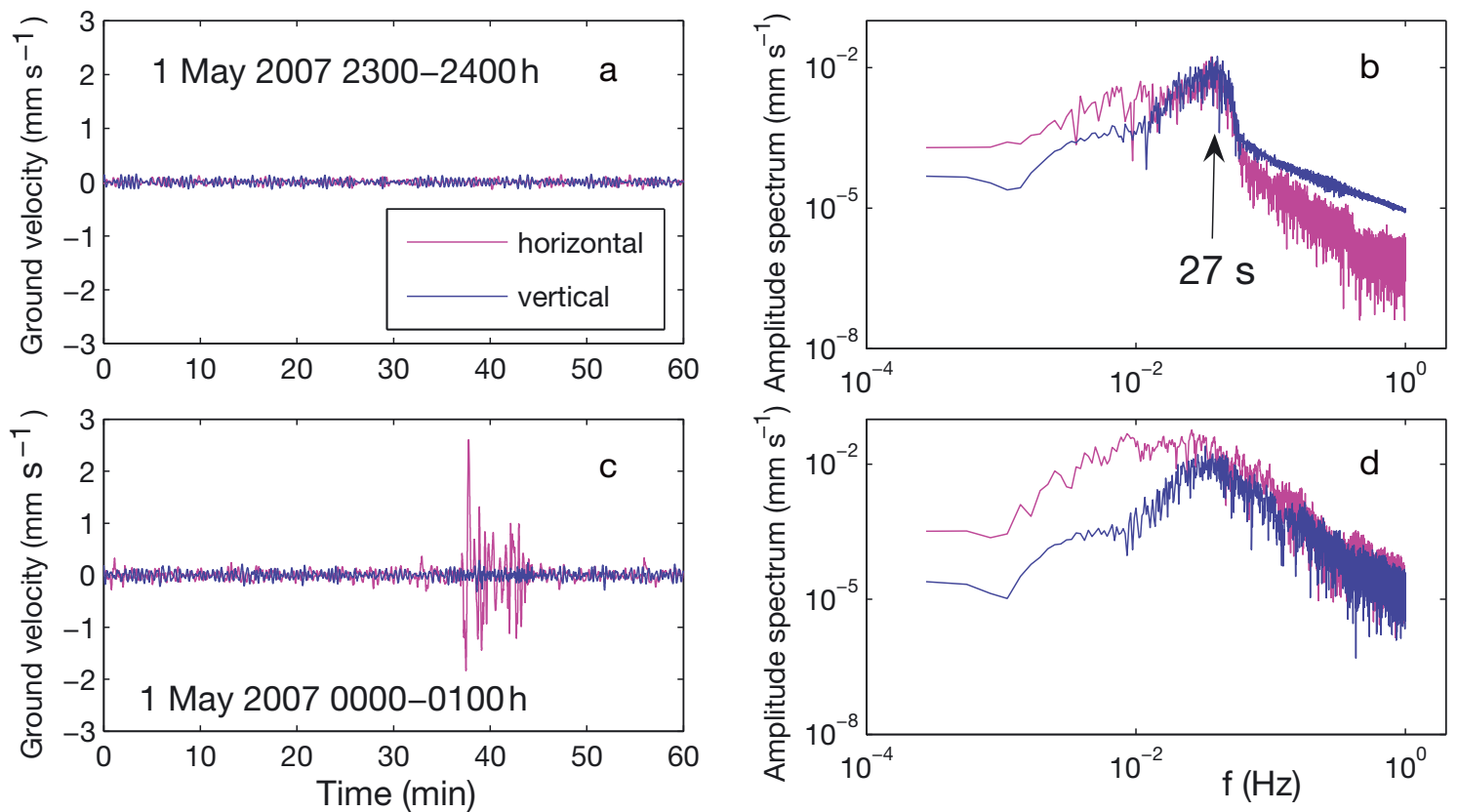

Fig. 2. Comparison of two of the recordings at station 1, each lasting 1 hour, one without and the other with a LFB. (a, c) Ground velocity on the vertical and the north (horizontal) channels. (b, d) Amplitude spectra of the ground velocity. (a, b) An hour with no LFB. The vertical and horizontal components have similar amplitudes and a spectral peak at 27 s. (c, d) An hour containing an LFB, only visible on the horizontal channels. The amplitude spectrum of the horizontal channel shows a clear increase at low frequencies (period $>27 \mathrm{~s}$ ).

that this burst is a measurement artifact. Because LFBs only generate horizontal displacement, they were not recorded by the 16 short-period vertical seismometers.

In order to systematically detect the LFBs, we use data from station 2 (which has the fewest data gaps during the investigated time period) to compute the ratio, $R=\sigma_{\mathrm{h}} / \sigma_{\mathrm{v}}$, of the rms (standard deviation) of the first horizontal channel and the vertical channel. This is done in successive, nonoverlapping $2 \mathrm{~min}$ time windows. There is considerable heterogeneity in the temporal fluctuations of $R$ (Fig. 3). In particular, in $11 \%$ of cases $R>3$, a threshold value that does not occur more than $10^{-6}$ times by chance if the rms of the horizontal channel is distributed identically to the rms of the vertical channel (Fig. 3b). Anomalously large values of $R$, attributed to LFBs, are observed, according to a density decaying as a power law in $R^{-2.7}$. This decay suggests that the source amplitude could also be distributed according to a power law, which agrees with previous observations on the power-law distribution of lead sizes and deformation amplitude (Rothrock and Thorndike, 1984; Marsan and others, 2004; Weiss and Marsan, 2004). Since the distances to the sources are unknown, we unfortunately cannot estimate a magnitude equivalent. The LFB occurrences are well correlated in time, exhibiting a temporal clustering similar to that characterizing crustal earthquakes (Fig. 3c). This could be caused by (1) the temporal correlation of the loading process (e.g. wind forcing) and/or (2) mechanical interactions between sources, as with crustal earthquakes.

\section{Polarization}

The arrivals of LFBs are well polarized. For each station, we compute for a sliding 1 min time window (1) the angle, $\phi$, that maximizes the energy of $h(t)=a(t) \cos \phi+b(t) \sin \phi$, where $a(t)$ and $b(t)$ are the two horizontal channels, and (2) the ratio, $R=\sigma_{\mathrm{h}}^{\max } / \sigma_{\mathrm{v}}$, where $\sigma_{\mathrm{h}}^{\max }$ is the rms of the energy-maximizing $h(t)$. Here we use $1 \mathrm{~min}$ rather than the
2 min windows above, because the polarization becomes much weaker at $2 \mathrm{~min}$, possibly as a result of local wave reflections (e.g. due to the heterogeneity of the ice thickness) that mix up different phases. Knowing the three angles, $\phi_{1}$, $\phi_{2}$ and $\phi_{3}$, that, for a given 1 min long window, maximize the horizontal energies, $h_{1}, h_{2}$ and $h_{3}$, we compute the differences $\phi_{i}-\phi_{j}$ for the three pairs of stations. For a well-polarized incoming wavetrain, these differences must be equal to the differences, $\theta_{i}-\theta_{j}$, in relative angles of the three stations, while for a poorly polarized signal they should randomly fluctuate. Figure 4 a shows the distribution of $\phi_{1}-$ $\phi_{2}$ for three intervals of ratio $R$ (no LFB, low-amplitude LFBs, high-amplitude LFBs). There is a clear tendency to become more polarized as $R$ increases, proving that the LFBs have a characteristic horizontal phase, either radial or transverse. Because the absolute angles, $\theta_{i}$, and the back-azimuth angles of the incoming waves (see below) are unknown, we cannot determine which of the two phases is actually observed.

\section{Source}

The distribution of the angles, $\phi$, for well-polarized LFBs (angle differences $\phi_{i}-\phi_{j}$ within $5^{\circ}$ of $\theta_{i}-\theta_{j}$ and $R>3$ ) shows that the LFBs have back-azimuth angles distributed over the whole interval $\left[0,180^{\circ}\right]$, with a more favorable direction (about $50-90^{\circ}$ for $\phi_{2}$; Fig. 4b). Although we cannot relate the angles, $\phi$, to absolute geographical directions, this proves that the LFB sources are distributed all around the seismic network and do not have a single geographical origin.

We applied seismic array methods to compute the angles at which the detected LFB wavetrains hit the seismic network. These beam-forming methods (e.g. Ringdal and Husebye, 1982) invert the absolute incident angle and the wave speed, based on the estimated time delays between the signals arriving at the three stations. We illustrate this for the LFB detected on 1 May 2007 at $0037 \mathrm{~h}$ (shown in Fig. 2c). 

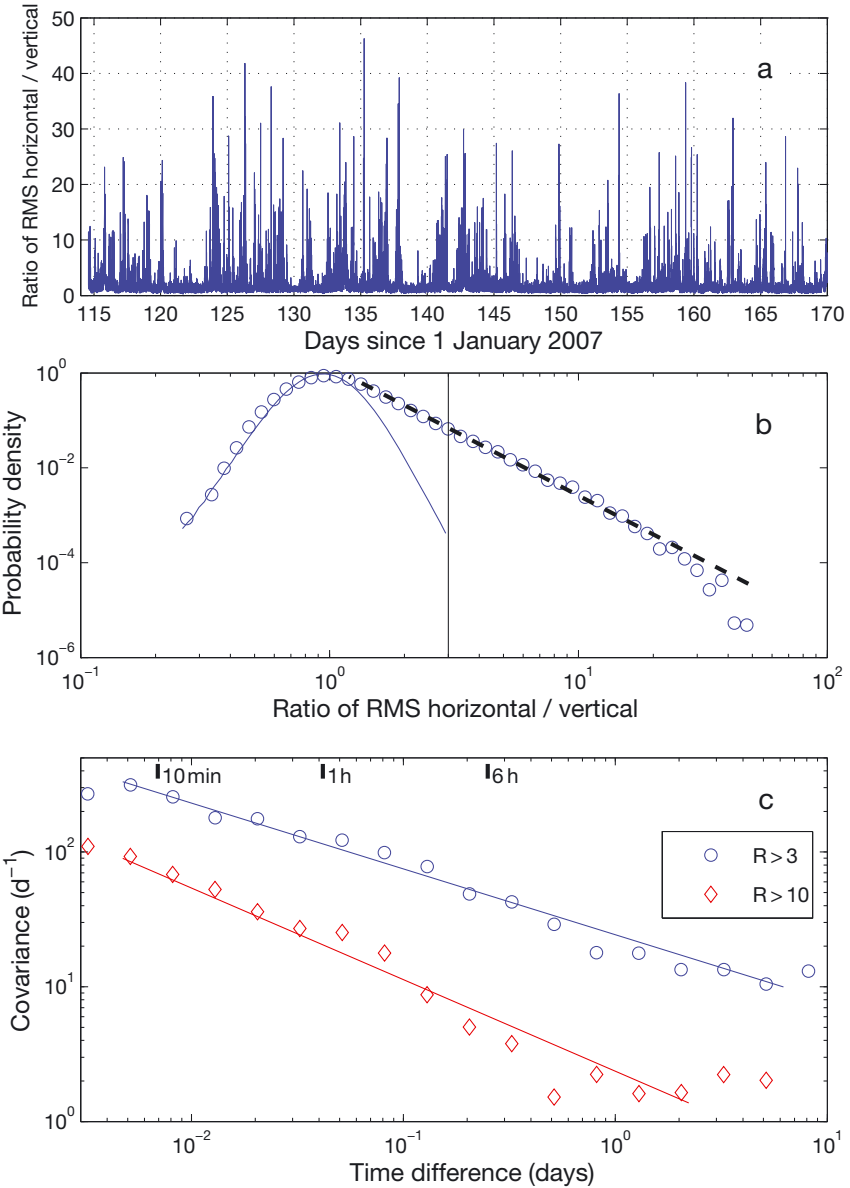

Fig. 3. (a) Ratio, $R=\sigma_{\mathrm{h}} / \sigma_{\mathrm{v}}$, of the rms of the first horizontal channel and the vertical channel of station 2 for successive 2 min long time windows. (b) Probability density of $R$. The density corresponding to the null hypothesis of no LFB (i.e. the horizontal channel only records the ice swell) is shown as a continuous curve, and explains only the $R<1$ values. The probability that $R>3$ (vertical line) occurs by chance (i.e. if the null hypothesis of no LFB were correct) is less than $10^{-6}$. For $R>1$, the density decays according to a power law, $R^{-2.7}$ (dashed line). (c) Temporal correlation for $R>3$ and $R>10$ events, showing a power-law decay in $\Delta t^{-0.48}$ and $\Delta t^{-0.68}$ respectively, for a time difference, $\Delta t$, in the range extending from $5 \mathrm{~min}$ to $2-5$ days.

For the $1 \mathrm{~min}$ time window starting at $0037 \mathrm{~h}$, we first compute the horizontal signals, $h(t)=a(t) \cos \phi+b(t) \sin \phi$, that maximize the energy, individually at the three stations (Fig. 5c). We obtain angle differences, $\phi_{i}-\phi_{j}$, within $4^{\circ}$ of $\theta_{i}-\theta_{j}$. The ratio, $R$, of the amplitudes of the computed horizontal, $h(t)$, and the vertical signals is 27.9, averaged over the three stations. This implies that this $1 \mathrm{~min}$ time window indeed contains the arrival of a well-polarized LFB. We compute the linear correlation coefficient for the three pairs of stations (Fig. $5 d$ ). The correlation is very high (99\%), and yields time lags of $1.05,0.82$ and $0.22 \mathrm{~s}$ for the three pairs. The accuracy on these estimates is low, as they are only stable $( \pm 0.12 \mathrm{~s})$ for $1 \mathrm{~min}$ time intervals starting within $30 \mathrm{~s}$ of $0037 \mathrm{~h}$. For later time intervals, other phases, including local reflections, are likely to be probed. Assuming an incident plane wave, frequency/wavenumber methods give a propagation speed of $440 \mathrm{~m} \mathrm{~s}^{-1}$ and a backazimuth angle as shown in Figure 6. This speed is much lower than the expected longitudinal plate (LP) or horizontally polarized shear $(\mathrm{SH})$ wave speeds (about 2800-3000 $\mathrm{m} \mathrm{s}^{-1}$
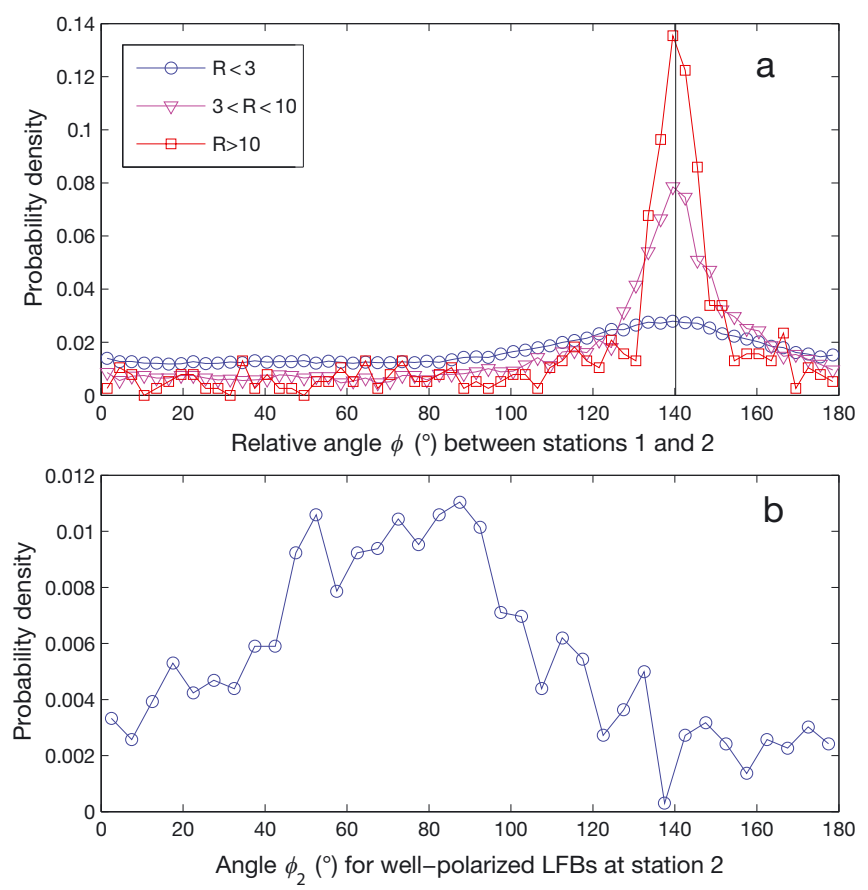

Fig. 4. (a) Probability density of the angle difference, $\phi=\phi_{1}-\phi_{2}$, between stations 1 and 2, where the angles $\phi_{1}$ and $\phi_{2}$ maximize the energy of the $1 \mathrm{~min}$ long horizontal wavetrains at the two stations. The density is plotted for three intervals of the ratio, $R$. For small values of $R(<3)$ the angle difference, $\phi$, is nearly uniformly distributed, hence is weakly polarized. For large values of $R(>10)$ there is a clearly favorable orientation that coincides with the angle difference $\theta_{1}-\theta_{2}=140.2^{\circ} \pm 1.9^{\circ}$ between the orientations of the first horizontal channels of both stations, which proves the strong polarization of the waves, either as longitudinal plate (LP) or horizontally polarized shear $(\mathrm{SH})$ phases. (b) Distribution of the angle $\phi_{2}$ that maximizes the energy of the horizontally polarized wavetrains at station 2 .

and 1600-1800 $\mathrm{m} \mathrm{s}^{-1}$, respectively; e.g. Hunkins, 1960). It is, however, much higher than the flexural wave speed in this frequency range $\left(\sim 24 \mathrm{~m} \mathrm{~s}^{-1}\right.$ for the group velocity at a period of $30 \mathrm{~s}$; Stein and others, 1998; Wadhams and Doble, 2009). We cannot relate the back-azimuth angle to a specific deformation transient as imaged by the hourly deformation map of Figure 6, obtained from the dispersion of the ice-tethered buoys operated by the Meteorological Institute of Hamburg. Given the typical error on ARGOS positioning, and the size of the triangle, the uncertainty on the deformation is $\sim 0.25 \times 10^{-3}$.

Generalizing this procedure to other LFBs highlights the very large dispersion found for the wave speed estimate, $v$. We selected clear LFB arrivals with the following criteria, using non-overlapping $1 \mathrm{~min}$ time windows: (1) a ratio $R>10$ when averaged over the three stations; (2) angle differences $\phi_{i}-\phi_{j}$ within $5^{\circ}$ of $\theta_{i}-\theta_{j}$; (3) visual selection of the portion of the time window that exhibits a clear correlation between the traces, along with visual inspection of the optimally time-shifted waveforms at the three stations in order to ensure the estimated time delays are meaningful. We obtained 63 such events, including the one discussed above (Figs 2c and 5). A least-squares fit of the absolute value of the time delays between stations 1 and 2, a function of $\phi_{1}$, gives $v=1090 \pm 380 \mathrm{~m} \mathrm{~s}^{-1}$ (Fig. 7). The large uncertainty in the estimate of $v$ is due to the dispersion of the values of the 

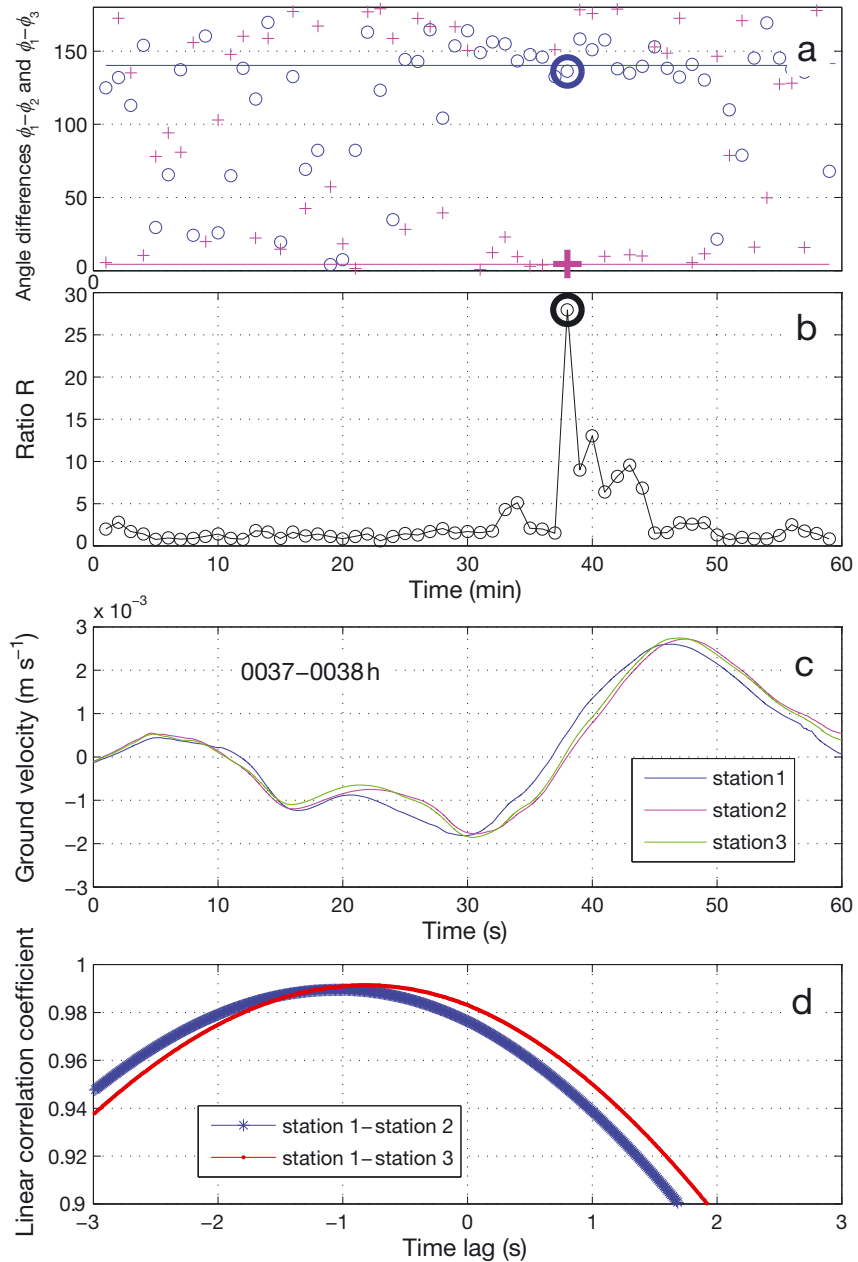

Fig. 5. Estimation of the incident angle of a LFB. (a) Difference in relative angles, $\phi_{1}-\phi_{2}$ and $\phi_{1}-\phi_{3}$, in degrees and (b) averaged ratio, $R$, for $601 \mathrm{~min}$ long time windows, on 1 May 2007, $0000-0100 \mathrm{~h}$. The 37 th minute is characterized by a well-polarized horizontal signal $\left(\phi_{1}-\phi_{2}\right.$ and $\phi_{1}-\phi_{3}$ close to the expected $\theta_{1}-\theta_{2}$ and $\theta_{1}-\theta_{3}$ as shown by the horizontal lines) with a high horizontal amplitude compared to the vertical signal (ratio $R=27.9$ ). We interpret these features as the arrival of a LFB. (c) Reconstructed horizontal signals at the three stations, for this 37 th minute. (d) Linear correlation coefficient between stations 1 and 2, and stations 1 and 3. The correlation reaches a maximum for time lags of -1.05 and $-0.82 \mathrm{~s}$.

time delays for nearly identical values of angle $\phi_{1}$. Although the confidence for the estimate of $v$ is low, it suggests that the LFB wavetrains are $\mathrm{SH}$ rather than LP waves. The attenuation coefficient can also be estimated as $0.0011 \mathrm{~dB} \mathrm{~m}^{-1}$, but with a large uncertainty $(76 \%$; Fig. $7 \mathrm{~b})$. The uncertainty comes from two main contributions: errors in the estimates of the time delays and uncertainty in the least-squares fit. Using the extreme values of 0.00024 and $0.0018 \mathrm{~dB} \mathrm{~m}^{-1}$, a tenfold absorption loss is equivalent to a propagation length ranging between 11 and $83 \mathrm{~km}$. Linearly extrapolating the log-log plot of Hunkins (1960) of the apparent frequency of LP waves vs distance (his fig. 1) to low frequencies, we find that these waves could propagate over distances of $\sim 50 \mathrm{~km}$ at a period of $50 \mathrm{~s}$. SH waves are expected to travel over longer distances as, contrary to LP waves, this is not a leaky mode; such long distances are thus coherent with our findings. This shows that the source is potentially located several tens of kilometers away from the seismometers.

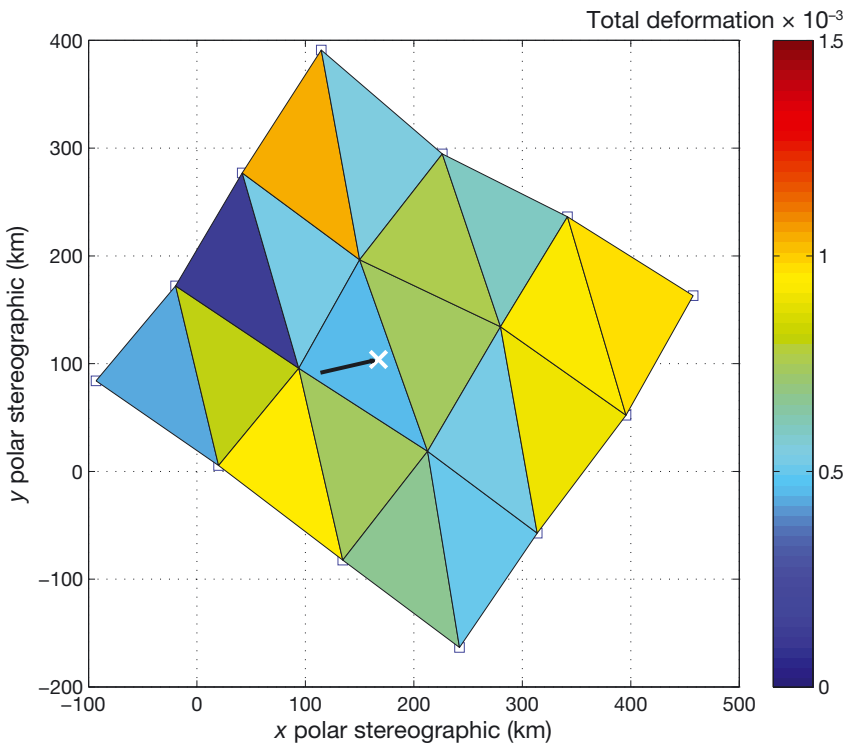

Fig. 6. Map showing the hourly total deformation on 1 May 2007, 0000-0100 h, around the seismic network (plotted as a white cross at the center of the map), as deduced from an array of 16 ice-tethered buoys. The estimated back-azimuth angle for the LFB detected at $0037 \mathrm{~h}$ is shown as a black line pointing from the seismic network to the direction of the source.
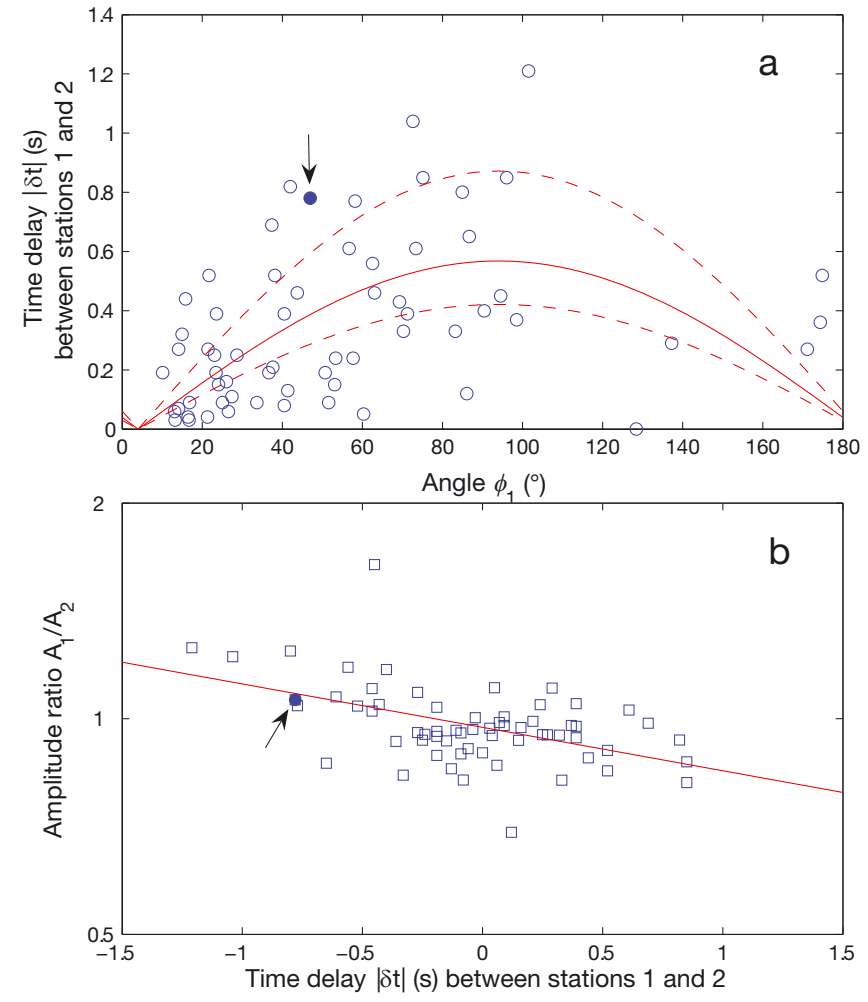

Fig. 7. (a) Distribution of the time delay between stations 1 and 2 vs the polarization angle, $\phi_{1}$, at station 1 , for the 63 selected clear LFB arrivals. The best fit (continuous curve) gives $v=1090 \mathrm{~m} \mathrm{~s}^{-1}$, with a standard deviation of $380 \mathrm{~m} \mathrm{~s}^{-1}$ (dashed curves). (b) Attenuation in log scale of the LFB wavetrain amplitude between stations 1 and 2 as a function of the time delays, for the same 63 LFBs. The best log-linear fit (continuous line) gives an attenuation of $0.0011 \mathrm{~dB} \mathrm{~m}^{-1}$. The two data points shown by arrows correspond to the 1 min long time window containing the LFB of Figure 5. 

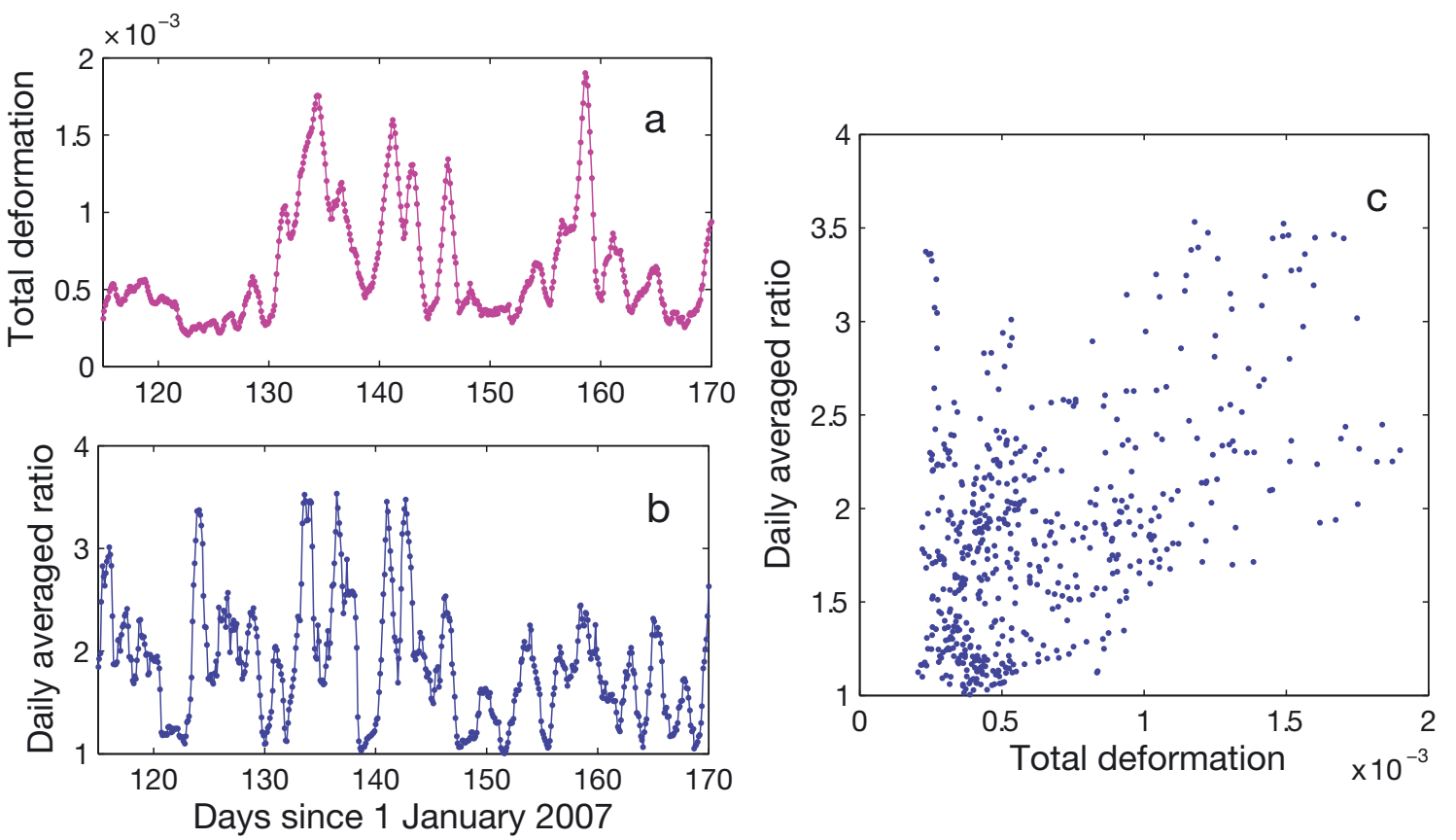

Fig. 8. Comparison of (a) the total deformation smoothed at 1 day for a $\sim 400 \mathrm{~km}$ triangle containing the seismic network with (b) the daily averaged ratio, $R$. (c) Daily averaged ratio vs total deformation, showing the correlation between the two.

Finally, in Figure 8, we compare the daily averaged ratio, $R$, to the daily total deformation, $\epsilon_{\text {tot, }}$ of a triangle of ice-tethered buoys operated by the Meteorological Institute of Hamburg. The total deformation is defined as $\epsilon_{\text {tot }}=\sqrt{\epsilon_{0}^{2}+\epsilon_{\text {shear }}^{2}}$, where $\epsilon_{0}$ is the divergence and $\epsilon_{\text {shear }}$ is the shear strain. This triangle is $\sim 400 \mathrm{~km} \times 400 \mathrm{~km} \times 570 \mathrm{~km}$ and includes the Tara drifting station. The linear correlation coefficient between $R$ and $\epsilon_{\text {tot }}$ is $49 \%$. Values of $51 \%$ and $10 \%$, respectively, are obtained using the shear or the divergence, rather than the total deformation. This significant correlation points to a possible origin for the LFBs, related to mechanical deformation of the sea-ice cover caused by shearing along leads at regional scales. The fact that the correlation is low suggests that only part of the ice-cover deformation at the scale of this triangle of buoys causes LFBs or perhaps that not all the related LFBs were detected by our network.

\section{DISCUSSION AND CONCLUSIONS}

The occurrences of LFBs are correlated in time (Fig. 3), with many distinct arrivals over hours, but can also be absent for long periods of time (hours to days). A key characteristic is that they correspond to horizontally polarized wavetrains. Several lines of evidence suggest that they are SH rather than LP waves. These modes do not leak into the water column. As such, they are not expected to generate any noise in the water, which could explain why they have gone unnoticed in ambient-noise studies based on hydrophone recordings. Hydrophones typically have a $1 \mathrm{~Hz}$ cut-off, which could also explain why they do not detect LFBs. However, the 16 short-period $(1 \mathrm{~Hz})$ seismometers deployed alongside the broadband seismometers used in this study recorded the energetic ice swell very clearly, although this signal has a characteristic period ranging between 20 and 30 s. As LFBs have horizontal displacement amplitudes even larger than those caused by the ice swell, they should be detectable by $1 \mathrm{~Hz}$ instruments if these instruments measure horizontal displacements. The fact that hydrophone studies have never noticed LFBs therefore suggests that LFB wavetrains do not propagate in the water column. Moreover, because $\mathrm{SH}$ waves can propagate over long distances in the ice without being strongly attenuated, we further propose that their sources are remote. They could be related to episodic shear (rather than convergent or divergent) deformation along regional-scale leads, as suggested by the $51 \%$ correlation between regional shear deformation and $R$, a proxy for LFB occurrences. SH modes are preferentially excited by such shear displacements. Assuming a typical corner frequency of $1 / 30$ s (Fig. 2d) and a rupture speed ranging between 0.6 and 0.9 times the bulk shear wave velocity (Madariaga, 1976) gives a typical rupture length of 20-30 km (Savage, 1972). Other seismological experiments, conducted with the same equipment on fast ice at Storfjord, Svalbard, and on Baltic sea ice, did not find any LFBs, which are therefore likely to be caused by large-scale deformation episodes. Given the 60 s cut-off of our instruments, the low-frequency content of these wavetrains could be significant at even longer periods than the dominant 30-100 s we find (Fig. 2d).

While satellite measurements allow us to map the kinematic structures that are responsible for the regional deformation of the Arctic sea-ice cover, much less is known about the dynamical aspects: do the regional-scale leads deform continuously, or do they exhibit an intermittent behavior, in the same way as crustal faults? This observation therefore raises the possibility of investigating these processes with good temporal sampling, to complement satellite imagery that can locate the regional-scale leads, but with a temporal precision limited to a few days.

\section{ACKNOWLEDGEMENTS}

This research was funded by the EU FP6 DAMOCLES project. We thank the crew at Tara, paticularly J. Festy and 
H. Le Goff, for instrumental maintenance, and B. Brummer of the Meteorological Institute of Hamburg for the ice-tethered buoy network data. We also thank E. Brossier at Vagabond and Institut Paul-Émile Victor for seismological data acquired during a 2005 field campaign at Storfjord, Svalbard that allowed comparison with the 2007 Tara data. Constructive reviews by two anonymous reviewers helped to clarify this paper.

\section{REFERENCES}

Crary, A.P. 1955. A brief study of ice tremors. Bull. Seismol. Soc. Am., 45(1), 1-9.

Dosso, S.E., G.J. Heard and M. Vinnins. 2002. Source bearing estimation in the Arctic Ocean using ice-mounted geophones. J. Acous. Soc. Am., 112(6), 2721-2734.

Dudko, Y.V. 1999. Analysis of seismo-acoustic emission from ice fracturing events during SIMI'94. (PhD thesis, Massachusetts Institute of Technology.)

Dudko, Y.V., H. Schmidt, K. von der Heydt and E.K. Scheer. 1998. Edge wave observation using remote seismoacoustic sensing of ice events in the Arctic. J. Geophys. Res., 103(C10), 21,775-21,781.

Dugan, J.P., R.L. DiMarco and W.W. Martin. 1992. Low-frequency vibrational motion of Arctic pack ice. J. Geophys. Res., 97(C4), 5381-5388.

Gascard, J.-C. and 25 others. 2008. Exploring Arctic transpolar drift during dramatic sea ice retreat. Eos, 89(3), 21-23.

Greening, M.V. and P. Zakarauskas. 1994. Pressure ridging spectrum level and a proposed origin of the infrasonic peak in arctic ambient noise spectra. J. Acous. Soc. Am., 95(2), $791-797$.

Hunkins, K. 1960. Seismic studies of sea ice. J. Geophys. Res., 65(10), 3459-3472.

Hunkins, K. 1962. Waves on the Arctic ocean. J. Geophys. Res., 67(6), 2477-2489.

LeSchack, L.A. and R.A. Haubrich. 1964. Observations of waves on an ice-covered ocean. J. Geophys. Res., 69(18), 3815-3821.
Madariaga, R. 1976. Dynamics of an expanding circular fault. Bull. Seismol. Soc. Am., 66(3), 639-666.

Makris, N.C. and I. Dyer. 1986. Environmental correlates of pack ice noise. J. Acous. Soc. Am., 79(5), 1434-1440.

Marsan, D., H. Stern, R. Lindsay and J. Weiss. 2004. Scale dependence and localization of the deformation of Arctic sea ice. Phys. Rev. Lett., 93(17), 178501. (10.1103/PhysRevLett.93.178501.)

Milne, A.R. and J.H. Ganton. 1964. Ambient noise under Arctic-sea ice. J. Acous. Soc. Am., 36(5), 855-863.

Milne, A.R., J.H. Ganton and D.J. McMillin. 1967. Ambient noise under sea ice and further measurements of wind and temperature dependence. J. Acous. Soc. Am., 41(2), 525-528..

Ringdal, F. and E.S. Husebye. 1982. Application of arrays in the detection, location, and identification of seismic events. Bull. Seismol. Soc. Am., 72(6B), S201-S224.

Rothrock, D.A. and A.S. Thorndike. 1984. Measuring the sea ice floe size distribution. J. Geophys. Res., 89(C4), 6477-6486.

Savage, J.C. 1972. Relation of corner frequency to fault dimensions. J. Geophys. Res., 77(20), 3788-3795.

Squire, V.A. 2007. Of ocean waves and sea-ice revisited. Cold Reg. Sci. Technol., 49(2), 110-133.

Squire, V.A., G.L. Vaughan and L.G. Bennetts. 2009. Ocean surface wave evolvement in the Arctic Basin. Geophys. Res. Lett., 36(22), L22502. (10.1029/2009GL040676.)

Stein, P.J. 1988. Interpretation of a few ice event transients. J. Acous. Soc. Am., 83(2), 617-622.

Stein, P.J., S.E. Euerle and J.C. Parinella. 1998. Inversion of pack ice elastic wave data to obtain ice physical properties. J. Geophys. Res., 103(C10), 21,783-21,793.

Wadhams, P. and M.J. Doble. 2009. Sea ice thickness measurement using episodic infragravity waves from distant storms. Cold Reg. Sci. Technol., 56(2-3), 98-101.

Weiss, J. and D. Marsan. 2004. Scale properties of sea ice deformation and fracturing. C. R. Phys., 5(7), 735-751.

Xie, Y. and D.M. Farmer. 1994. Seismic-acoustic sensing of sea ice wave mechanical properties. J. Geophys. Res., 99(C4), 7771-7786.

Yang, T.C. and G.R. Giellis. 1994. Experimental characterization of elastic waves in a floating ice sheet. J. Acous. Soc. Am., 96(5), 2993-3009. 